(Aus der Grossherzog1. Universitäts-Frauenklinik $z u$ Giessen. Director: Geh. Med.-Rath Prof. Dr. Pfannenstiel.)

\title{
Keilresection des Corpus uteri wegen chronischer Metritis, insbesondere bei der Prolapsoperation.
}

\author{
Von
}

\section{Dr. Franz Cohn,}

Assistenzarzt der Ḱlinik.

Bei denjenigen Formen des Prolapses, welche der Schädigung des Genitalapparats durch vorangegangene, meist vielfache Geburtsarbeit ihre Entstehung verdanken, finden wir sehr häufig das Bestehen höherer Grade von Metritis. Es ist dies keineswegs ein irrelevanter Nebenbefund, der erst bei der Vornahme der gynäkologischen Untersuchung entdeckt wird, vielmehr lenkt schon von vornherein eine grosse Zahl intensiver Klagen über Druckgefühle und Menstruationsanomalieen, welche von den Patientinnen meist auf den von ihnen wahrgenommenen Prolaps bezogen werden, die Aufmerksamkeit des Arztes auf die Veränderungen des Uterus.

Auch unter den Gynäkologen selbst sind die Ansichten über den ätiologischen Zusammenhang von Prolaps und chronischer Vetro-Endometritis noch keineswegs übereinstimmend. Manche Autoren verallgemeinern die allerdings unbestreitbare ödematöse Anschwellung des vorgefallenen Uterus zu sehr und stellen eine echte Hypertrophie des chronisch gereizten Organs in Abrede. Nach ibnen müsste es sich also um einen vorübergebenden Zustand handeln und der Uterus nach Beseitigung des Prolapses stets seine normale Beschaffenheit wiedergewinnen.

Andere sehen in einer primären Retroversio-flexio die Ursache sowohl für den Prolaps, als auch für einen durch Staung bedingten chronisch-hyperplastischen Zustand des Uterus, weJcher dem anatomischen Bilde der chronischen Endometritis und Metritis 
entspricht (Küstner). Nach dieser Anschauung müsste die Correctur der Lageveränderung stets eine Rückbildung des geschwellten Organs zur Folge haben; höchstens werde bei höheren Graden von Endometritis corporis die Entfernung der ödematösen Schleimhaut durch Ausschabung nothwendig.

Endlich besteht auch noch die umgekehrte Meinung, dass nämlich eine primär auf chronisch-entzündlicher Basis entstandene Metritis durch die vermehrte Schwere des Uteruskörpers die Retroflexion desselben und eine Senkung von Uterus und Scheide zur Folge habe.

Die Beantwortung der Frage nach dem ätiologischen Zusammenhang zwischen dem Prolaps und der ihn begleitenden Metritis ist nun nicht bloss von theoretischem Interesse, sondern von grosser Bedeutang für die einzuschlagende Behandlung, namentlich für die operative Therapie. Wer auf dem Boden der Anschauung steht, dass die metritisch-endometritische Veränderung des Uterus nur einen passageren, durch die bei Prolaps oder Retroflexio bedingte Stauung verursachten Zustand darstellt, welcher nach Beseitigung der Lageanomalie in einen anatomisch und functionell normalen Status zurückgeht, der wird sich damit begnügen, die Retroflexio oder den Prolaps zu beseitigen und wird erwarten, die Patientin damit auch von ihren metritischen Beschwerden geheilt zu haben. Höchstens wird er noch durch eine Abrasio der erkrankten Uterusschleimhaut zu Leibe gehen.

Wer indessen die den Prolaps begleitende Metritis nicht bloss als eine wieder reparable Folgeerscheinung ansieht, sondern ihr eine mehr selbständige Rolle einräumt, der wird, natürlich von den Resultaten seiner Therapie geleitet, bestrebt sein, die durch die Metritis an sich verursachten Erscheinungen diagnostisch und therapeutisch von den Symptomen der Retroflexio und des Prolapses abzugrenzen.

Die Pfannenstiel'sche Klinik vertritt seit langem den Standpunkt, dass die chronische Metritis des Uteruskörpers, auch wenn sie mit Descensus oder Retroflexio uteri verbunden ist, ätiologisch als eine selbständige Erkrankung anzusehen und therapeutisch als solche gesondert zu berüeksichtigen ist. Die ehronische fibromatöse Verdickung der Uteruswand, die als echte Metritis fibromatosa hyperplastica $z u$ bezeichnen ist, und die wir namentlich bei multiparen, mit mehr oder minder hochgradigem Descensus oder Prolaps der Schejde behafteten Franen antreffen, 
ist wohl zu unterscheiden von der nur vorübergehenden und wieder reparablen ödematösen Anschwellung des prolabirten oder descentirten Uterus. Bei den chronisch verdickten Uteris, die auch nach Reposition des Prolapses dauernd ihre abnorme Grösse beibehalten, haben wir uns durch zahlreiche mikroskopische Untersuchungen davon überzeugt, dass es sich hier um wahre hyperplastische Vorgänge in der Uteruswandung, und zwar hauptsächlich im Bindegewebe handelt. Diese dauernde und in ihrer Entstehung oft noch vor die Ausbildung des Prolapses zurückzudatirende Veränderung des Uterus ist nicht als eine Folge des Prolapses anzusehen. Vielmehr hat sie mit der Senkung der Genitalien höchstens die Aetiologie in weitem Sinne gemeinsam, die in den vorausgegangenen Geburtstraumen mit ihren Folgezuständen zu suchen jst. Wenn indes die Schädigungen der vorangegangenen Geburten sich isolirt entweder nur auf eine Erschlaffung des Bandapparates oder durch bakteriotoxische oder chronisch-hyperämisirende Reize allein auf die Schleimhaut und die Wand des Uterus erstrecken, kann es natürlich auch zu einem Prolaps ohne Metritis oder zu einer chronisch-hyperplastischen Metro-Endometritis ohne Prolaps kommen. Dass secundär dann die Schwerezunahme des Uterus durch die Metritis die Ausbildung eines Prolapses begünstigen kann, ist nicht zu leugnen.

In der Frage des Zusammenhanges von Retroflexio uteri und chronischer Metritis ist die Stellungnahme der Pfannenstiel'schen Klinik, wie überhaupt gegenüber der Bedeutung der Retroflexio, sehr skeptisch. Dass die Retroflexio an sich durch Veränderung der Blutversorgung eine hochgradige Stauung im Uteruskörper und dadurch eine fibromatöse Hyperplasie desselben hervorrufen könne, erscheint keineswegs bewiesen. Dass zahlreiche verdickte retroflectirte Uteri nach Aufrichtung und orthopädischer Fixirung in Anteflexionsstellung ihre abnorme Grösse nicht im geringsten einbüssen, spräche jedenfalls dagegen; es müssten denn in allen diesen Fällen die angeblich durch Stauung verursachten Veränderungen schon völlig irreparabel geworden sein.

Dass ein metritisch verdicktes Corpus sich auf einem schlanken Halse sehwerer balanciren kann, als ein normal grosses, and in Folge dessen eher zu einer mobilen Retroflexio disponirt ist, kann man sich leichter vorstellen. Dies wäre dann der umgekehrte ätiologische Zusammenhang zwischen Retroflexio und Metritis.

Von der Anschauung ausgehend, dass eine mobile Retroflexio eines sonst gesunden Uterus, die nicht durch entzündliche Processe 
in der Umgebung des Uterus somplicirt ist, überhaupt keine Beschwerden macht ${ }^{1}$ ), haben wir gefunden, dass in den relativ seltenen Fällen von mobiler Retroflexio, in denen im Uterus selbst und nicht im Nervensystem oder in allgemeinen Schwächezuständen des Organismus die Ursache für die sogenannten Retroflexionsbeschwerden zu suchen ist, die complicirende Metritis für diese Beschwerden verantwortlich gemacht werden kann. Während ein normal grosses bewegliches Corpus in Retroflexionsstellung auf das Rectum und die hintere Beckenwand höchstens denselben Druck ausüben kann, den es in Normallage auf die Blase ausübt, sind Druckbeschwerden nach hinten, die durch ein abnorm schweres retroflectirtes Uteruscorpus verursacht werden, sehr wohl anzunehmen und auch zu beweisen. Das sind die Fälle, in denen das lastende Organgefühl des vergrösserten Uterus von den Patientinnen oft sehr präcis, meist ins Reetum, localisirt wird, in denen die Bewegungen des hin- und herfallenden Uterus deutlich wabrgenommen werden, und in denen nach Lagecorrectur des metritischen Uterus die Druckgefühle hinten verschwinden, dafür aber häufig nach anderer Richtung hin empfunden werden. Hingegen erlebt man nicht selten bei Retroflexio kleiner, nicht metritischer Uteruskörper die Enttäuschung, nach der Richtigstellung des Uterus die Kreuzschmerzen und ähnliche "Retroflexionsbeschwerden" unverändert fortbestehen zu sehen; dieses eben sind die Fälle, in denen die Beschwerden nicht durch die mobile Retroflexio, sondern durch Neurasthenie oder allgemeine Schwächezustände bedingt waren, und in denen der Eingriff der Lagecorrectur in seiner sonst häufig so prompten Suggestivwirkung versagt hat.

Ausser den durch die chronische Metritis verursachten Druckbeschwerden, die durch das Hinzutreten eines Prolapses noch mehr, bis zur Unerträglichkeit gesteigert werden können, sind es die Blutungen, welche die Patientinnen mit chronischer Metritis oft noch mehr als die Druckschmerzen beunruhigen, und die in Folge dessen in letzter Zeit ein gesteigertes therapeutisches Interesse erweckt haben.

Bei den Myomblutungen sind die dauernden Schädigungen des Herzens, die die Gefahr ernster Kreislaufstörungen in sich bergen

1) Pfannenstiel, Zur Discussion über die Behandlung der Falschlagen vơn Uterus und Scheide. Monatssehr. f. Geburtsh. und Gynäkologie. Bd. XVIII. 1903. 
und die Prognose einer bis zum Eintreten von Herzveränderungen hinausgeschobenen Operation von vornherein wesentlich verschlechtern, schon langst in ihrer Bedeutung gewürdigt worden. Immer mehr kommt man davon ab, in der Hoffnung auf das bald zu erwartende, in Wirklichkeit aber durch das Myom erheblich verzögerte Klimacterium, die Frauen bis zu der bekannten wachsbleichen Hautfarbe, bis zur Ausbildung eines typischen "Myomherzens" sich ausbluten zu lassen, anstatt rechtzeitig die operative Entfernung des Myoms vorzunehmen.

Dieselbe Stärke wie beim Myom können die Blutungen auch bei der chronischen Metritis erreichen, und sie ziehen dann natürlich auch dieselben Folgen nach sich wie die Myomblutungen. Wir haben unter unserem Material Metritis-Fälle gesehen, die bis auf's Aeusserste ausgeblutet die Klinik aufsuchten und bereits schwere Herzveränderungen aufwiesen.

Freilich findet man in chronisch-metritischen Uteris bei sorgfältiger Durchsuchung der verdickten Wand ab und zu auch kleine intramurale Myomknötchen in grösserer oder geringerer Anzahl; indess sind diese bei ihrer Kleinheit und ihrer von der Uterusmucosa entfernten Lage nicht für die Entstehung der Blutungen verantwortlich zu machen, sondern nur als Theilerscheinung der beträchtlichen hyperplastischen Wucherung aller Theile der Uteruswand anzusehen.

Die nicht seltenen Fälle hochgradiger Anämie und Erschöpfung in Folge der Blutungen bei chronischer Metritis, die wir zu beobachten Gelegenheit hatten, haben uns veranlasst, uns der Metritis-Frage diagnostisch und therapeutisch intensiv zu widmen. Sehon während seiner Wirksamkeit in Breslau hat Pfannenstiel in Folge des häufigens Versagens der Abrasio gerade bei den hochgradigen Fällen metritischer Blutungen in der Atmokausis ein Mittel zu ergreifen versucht, das einen harmlosen Ersatz für eine operative Therapie der Metritis-Blutungen zu bieten versprach ${ }^{1}$ ). Diese Erwartung hat sich nicht erfüllt. Trotz richtiger Anwendung der von Pincus angegebenen Technik gewährt die Atmokausis keinen sicheren Erfolg und kann andererseits unangenehme Complicationen zur Folge haben, die das Verfahren direct als gefährlich erscheinen lassen ${ }^{2}$ ). Wir haben daher die Atmokausis vollkommen

1) I achmann, Die Vaporisastion des Uterus. Münch. med. Wochenschrift 1901. No. 22 u. 23.

2) Pfannenstiel, Zur Frage der Atmokausis uteri. Honatsschr. für Geburtsh. u. Gynäk. Bd. XXIII. 1906. 
aufgegeben und haben seitdem alle ernsteren Metritis-Hällo, darunter auch einige früher mit Atmokausis erfolglos behandelte, der operativen Behandlung unterzogen. Bei incomplicirten Fällen hochgradiger Metritis mit starken Blutungen haben wir die vaginale Totalexstirpation des Cterus mit Erhaltung der Adnexe ausgeführt, z. B. von Januar 1906 an 9 mal. In Fällen, in denen durch complicirende Adnextumoren oder chronische Perityphlitis der abdominale Weg angezeigt war, haben wir mehrfach die supravaginale Amputation, einmal die abdominale Totalexstirpation des metritischen Uterus ausgeführt.

Von einem anderen Gesichtspunkte aus muss das einzuschlagende Operationsverfahren betrachtet werden, sobald zu der chronischen Metritis die Complication eines Prolapses hinzutritt, und dieser Combination begegnen wir in Folge der gemeinsamen Entstehungsursache beider Affectionen, der vorangegangenen häufigen Geburtstraumen, relativ oft. Hier muss die Operation zwei Indicationen gerecht werden: der (plastischen) Beseitigung des Prolapses und der definitiven Behebung der metritisch-endometritischen Beschwerden, d. h. der durch die abnorme Schwere des Uterus hervorgerufenen Druckgefühle, der durch die Schleimhaut- und Wandhypertrophie verursachten Blutungen und endlich des vom geschwellten Endometrium ausgehenden Fluors.

Als rationelle Prolapsoperation wird wohl jetzt überall eine der Fixationsmethoden des Uterus, verbunden mit Scheidendammplastik, ausgeführt. An der Pfannenstiel'sehen Klinik ist als das souveräne Verfahren seit langen Jahren die Vaginifixur des Uterus bei allen irgendwie erheblichen Descensus- und Prolapsformen im Gebrauch, ausnahmslos, sobald eine Cystocele besteht oder der Uterus selbst an der Senkung betheiligt ist. Gerade, dass der Uterus selber als Pelotte zur Prolapsplastik verwendet wird, ist der enorme Fortschritt der modernen vaginalen Fixationsmethoden. Die Ventrifixur des Uterus als Prolapsoperation haben wir gänzlich verlassen.

So einfach die Indicationsstellung beim incomplicirten Prolaps ist, in so grosse Verlegenheit geräth - man, wenn sich mit dem Prolaps eine chronische Metritis mit heftigen Blutungen und Druckbeschwerden combinirt. Begnügt man sich damit, gegen die MetroEndometritis mit einer Abrasio vorzugehen und den überdies durch seine Grösse und Schwere für die Plastik nicht sehr geeigneten Uterus vaginal oder anderwärts zu fixiren, so hat man das Risiko, 
dass die Abrasio, wie auch wir es mehrmals erleben mussten, erfolglos bleibt und die Blutungen andauern. Erneute Abrasionen haben dann meist nicht mehr Erfolg als die erste und sind ausserdem am vaginifixirten Uterus schwer mit Gründlichkeit anszuführen. Man steht dann vor der schweren Aufgabe, den fest fixirten Uterus nochmals operativ und dann radical in Angriff zu nehmen.

Geht man dagegen von vormherein radical gegen die chronische Metritis vor, indem man die vaginale Totalexstirpation des metritischen Uterus ausführt, so verliert man für die Prolapsplastik die unschätzbare Pelotte des Uterus, die einen sicheren Schutz gegen ein Recidiv der Cystocele abgiebt. Wir haben nach rationell ausgeführter Vaginifixur niemals ein Recidiv einer Cystocele gesehen, während wir in nicht seltenen Fällen nach Totalexstirpation des Uterus constatiren konnten, wie sich die descendirten, schlaffen und haltlosen Scheidenwände mit Cystocele und Rectocele selbst durch einen operativ stark verengten Introitus vorwölbten und zu hochgradigen Prolapsbeschwerden Veranlassung gaben.

Diesem Dilemma haben wir durch ein neues Operationsverfahren abzuhelfen gesucht, durch die Keilresection des Uteruskörpers. ${ }^{1}$ ) Die Methode soll den beiden Indicationen gerecht werden, für die Prolapsplastik eine vom Uterus gebildete Pelotte zu erhalten, andererseits aber durch Entfernung der Corpus-Schleimhaut die Blutungen definitiv $z u$ beseitigen und durch Verkleinerung des Uteruskörpers denselben auf seine normale Grösse und Schwere $7 u$ reduciren. Derartige Operationen hat $P$ fannenstiel schon einige Male während seiner Breslauer Thätigkeit ausgeführt, zum Theil nur aus der Veranlassung, weil der zu grosse und zu schwere metritische Uterus trotz Amputation der Portio nicht in die Plastik der Vaginifixur passen wollte. Planmässig haben wir die Operation erst in Giessen ausgeführt; der erste derartige Fall wurde im November 1903 operirt. Dann haben wir uns nach längerer Pause, in der die vaginale Totalexstirpation überwiegte, seit Anfang 1906 wieder der Keilresection zugewandt und seitdem dieses Verfahren in 29 Fällen, zusammen also in Giessen 30 mal angewendet. Pfannenstiel hat bereits auf dem 15. internationalen Congress

1) Die von Dührssen angegebene Excisio vaginalis mucosae uteri entspringt anderen Indicationen nnd ist für die chronisch-metritische Verdickung des Uterus nicht vorwendbar. 
in Lissabon die Methode kurz publicirt. ${ }^{1}$ ) Die guten Resultate der Operationen ermuthigen uns, die Methode jetzt ausführlicher der Oeffentlichkeit zu unterbreiten.

Die Technik der Operation, welche im Grunde ein Mittelding zwischen Vaginifixur und vaginaler Totalexstirpation darstellt, bietet keine: besonderen Schwierigkeiten. Der Anfang der Operation gestaltet sich ganz wie bei der an der Pfannenstielschen Klinik seit Langem üblichen Methode der vaginalen Fixur. Wir beginnen mit der Excision eines ovalen Lappens aus der vorderen $V$ aginalwand, dicht hinter dem Urethralwulst anfangend und bis zur Portio herabreichend. Bei besonders hochgradig verdicktem Corpus fügen wir, um den Zugang weiter zu machen, noch einen queren Schnitt vor der Portio hinzu, so dass im Ganzen eine $\perp$-förmige Schnittfigur entsteht. Die Blase wird von den Rändern der Colporrhaphieschnitte losgelöst, indem zuerst dicht an dem durch Klemmen angespannten Wundrande mit senkrecht aufgestelltem Messer das Blase und Scheide verbindende subvaginale. Bindegewebe vorsichtig durchtrennt und darauf die Blase weiter stumpf mit Unterminirung der Scheidenränder abgeschoben wird. Man bleibt durch diese erst scharfe, dann stumple Ablösung am sichersten in der richtigen Trennungsschicht und vermeidet so die unangenehme Verletzung der zahlreichen venösen Gefässe der Blasen- und Scheidenwand. Hierauf wird unter medialer Anspannung der Blase diese mit der Scheere ron der Cervix medial abgetrennt und mit dem Finger in der Mitte etwas nach oben geschoben, was stets leicht gelingt. Durch Einsetzen eines kleinen Speculum in die nun median entstandene Lücke werden jetzt beiderseits die seitlichen, die Gefässe enthaltenden bindegewebigen Verbindungen zwischen Blase und Cervix angespannt, die wir als die "seitlichen Blasenpfeiler" (Ligamenta cervico-vesicalia) bezeichnen. Diese werden jederseits zwischen zwei nach hinten convergirenden Klemmen durchgeschnitten; ein Mitfassen des Ureters ist dabei unter normalen Verhältnissen ausgeschlossen und uns nie vorgekommen. Die Klemmen werden sodann beiderseits, nach der Blase und nach der Cervix zu, durch Ligaturen ersetzt. Nach Durehtrennung ihrer bindegewebigen Verbindungen mit der Cervix

1) Pfannenstiel, Zur. Therapie der chronischen Endometritis. XV. Congrès international de médecine. Lisbonne. Avril 1906. Section XIII: Obstétrique. 
rückt die Blase schon von selbst etwas nach oben und kann nun mit Leichtigkeit vollends in die Höhe bis über den Uterus hinauf geschoben und dort durch ein Speculum festgehalten werden. Nach querer oder Längsspaltung der sich nun präsentirenden vorderen Umschlagsfalte des Peritoneum wird jetzt der verdickte Uteruskörper mit Kugelzangen oder Muzeux'schen Zangen bis vor die Vulva hervorgeleitet, während die Portio nach hinten in die Kreuzbeinaushöhlung gestaucht wird. Bis hierher gleicht das Verfahren völlig der bei uns üblichen Vaginifixur-Methode. Ist die Portio stark hypertrophisch, die Cervikalschleimhaut durch seitliche Cervixrisse ektropionirt, so schicken wir der vorderen Colpotomie eine meist recht ausgiebige Amputation der Portio vorats.

Zur Resection eines medianen Keils aus dem metritisch verdickten Corpus werden nun zwei grosse sagittale symmetrische Schnitte über den Fundus angelegt, die an der Vorder- und Hinterwand des Uterus nach der Gegend des inneren Muttermundes zu convergiren. Die Grösse des zu excidirenden Keils variirt natürlich nach dem Grade der metritischen Verdickung des Uterus. Wir wählen das zu entfernende Stück in der Regel so gross, dass der übrigbleibende Rest reconstruirt ein dem normalen Uterus an Grösse entsprechendes Organ ergiebt, dass also jeder Seitentheil einer normalen Uterushälfte entspricht, wie wir sie von der medianen Spaltung des Uterus bei der Totalexstirpation zu sehen gewohnt sind. Dann fällt der excidirte Keil fast stets so gross aus, dass or die gesammte Corpus-Schleimhaut in sich enthält. Sollte an den seitlich restirenden Stücken noch ein Theil der Mucosa stehen geblieben sein, so excidiren wir diese noch nachträglich mit dem Messer.

Die nach der Excision des Keiles entstehende Blutung ist meist ziemlich beträchtlich; es spritzt aus zahlreichen kleineren Arterien. Grössere Uterinaäste werden indes, wenn man die Schnitte nicht tiefer als bis zum inneren Muttermund herabführt, meist nicht getroffen.

Durch Anziehen der seitlichen Uterusstücke lässt sich die Blutung wesentlich beschränken, wie wir dies ja auch von der vaginalen Totalexstirpation mit medianer Spaltung ber kennen. Eine weitere Verringerung des Rlutzuflusses lässt sich auch dadurch erzielen, dass man vor der Resection des Keils die uterinen Tubenenden mit der angrenzenden Ligamentpartie beiderseits abbindet, um die Anastomosen von der Spermatica her auszuschalten. 
Auf isolirte Versorgung der blutenden Gefässchen lassen wir uns nicht ein, sondern schreiten so schnell als möglich zur Neoformation des Uterus. Je rascher wir diese mit besserer Einübung der Technik ausgeführt haben, desto mehr haben wir auch den Blutverlust zu beschränken gelernt. Während der Vereinigung werden die noch nicht zusammengenähten Theile digital oder mit Tupfern comprimirt gehalten.

Die sagittal in der Medianlinie erfolgende Vereinigung der seitlichen. Theilstücke führen wir je nach der grösseren oder geringeren Dicke der metritischen Uteruswand entweder mit einer einzigen durchgreifenden Nahtetage oder einer versenkten und einer oberflächlichen Etage von Catgut-Knopfnäht aus, wobei wir auf die sorgfältige Adaptirung der Wundränder grossen Werth legen. Aus diesem Grunde wenden wir auch keine fortlaufende Naht an, die eine exacte Adaptirung selten zu Stande kommen lässt. Natürlich trägt auch möglichste Schärfe und Glattheit der Schnittflächen sehr viel zu einer glatten Vereinigung und Heilung bei. Als Nahtmaterial verwenden wir ausnahmslos Catgut und zwar vorwiegend Jodeatgut, seltener Cumoleatgut.

Dis Vernähung der seitlichen Theilstüeke zu einem neuen Uterus beginnen wir am untersten Punkte der Schnitte an der hinteren Uterusfläche, um, über den Fundus fortschreitend, vorn in der Gegend des inneren Mattermundes zu endigen. Die Anzahl der gelegten Knopfnähte beschränken wir, um Nekrosenbildung zu vermeiden, auf das möglichste, soweit es eine exacte Blutstillung zulässt. Wenn noch kleine Stichkanalblutungen bestehen; so haben wir die Nahtlinie extraperitoneal zu legen gesucht, indem wir den vorderen Rand der eröffneten Peritonealfalte auf die Hinterwand des Uterus mit einer oder zwei Nähten fixirt haben.

Der jetzt neugebildete und normal grosse Uterus wird nun nach der bei uns üblichen Vaginifixirmethode mit zwei SilkwormNähten fest an die Vagina angeheftet, indem wir die Fixationsfäden ungefähr in der Höhe der Ansatzstellen der Ligamenta rotunda durch die Vorderfläche des Uterus führen und sie dicht unterhalb des Urethralwulstes aus dem Scheidenrande herausleiten. Unter exacter Adaptirung der Scheidenwundränder werden die Nähte sogleich geknöpft, während der Uterus durch Stauchen der Portio nach hinten in der gestreckten Lage gehalten wird, die ihm die Vaginifixur aufnöthigen soll. Sodann erfolgt die Vernähung der vorderen Colporrhaphie, bei welcher der Uterus ebenfalls weiter 
in situ gestreckt erhalten und nicht wieder an der Portio, vor die Vulva gezogen wird. Den Schluss der Operation bildet eine mehr oder minder ausgiebige hintere Colporrhaphie und Dammplastik.

Nach Beendigung der Operation haben wir dieselben plastischen Verhältnisse vor uns, wie nach der Vaginifixation eines normalen Uterus.

Wir haben unter Beseitigung der metritischen Verdickung des Uterus für die Prolapsplastik eine Pelotte geschaffen, die einem normalen Uterus entspricht und bei späterer Untersuchung von einem solchen nicht $\mathrm{zu}$ unterscheiden ist.

Wir haben die Verstümmelung der totalen Uterusexstirpation vermieden mit ibren unangenehmen Folgen für das plastische Resultat und den allgemeinen Ausfallserscheinungen, wie sie nach radikaler Entfernung der Gebärmutter beobachtet worden sind. Allerdings ist mit unserer Methode natürlich der volle Verlust der Conceptionsfähigkeit gegeben. Jedoch kommt man eben nur bei älteren und vielgebärenden Frauen in die Lage, wegen hochgradiger Metritis mit Prolaps die Keilresection des Uterus auszuführen.

Unter unseren 30 Fällen standen nur 4 in der ersten Hälfte des 4. Lebensjahrzehnts, 7 in der zweiten Hälfte und 19 jenseits des 40. Lebensjahres. Alle waren mehrfache Mütter mit Ausnahme zweier Patientinnen, deren metritischer Uterus zahlreiche intramurale Myome enthielt und für eine Conception nicht viel Aussicht bot.

Die eine dieser beiden Erauen war seit langem steril verheirathet, die andere hatte eine Geburt hinter sich (Fall 19 und 15).

Trotz des Verlustes der Conceptionsfähigkeit ist den Patientinnen doch das Bewusstsein, nicht durch völlige Herausnahme der Gebärmutter verstümmelt zu sein, von grossem Werthe.

Es ist gerade dies von einer Anzahl unserer Fälle ausdrücklich und dankbar anerkannt worden.

Es ist sogar möglich, dass durch Zurücklassung eines kleinen Mucosa-Restes im unteren Theile des Corpus die Menstruation erhalten bleibt, was für das psychische Gleichgewicht der Operirten oft als sehr wünschenswerth erscheint.

Bei einigen Operationen haben wir absichtlich den unteren Theil der Corpusmucosa geschont um bei der Neuformung des Uterus ein kleines, mit Schleimhaut ausgekleidetes Cavum zu construiren und so den Patientinnen den Schein eines functionirenden Organs zu gewähren. 
Unter unseren Fällen haben wir 9 mal nach der Operation die Periode wieder auftreten sehen, meist in regelmässigem Typus, stets bedeutend schwächer als vor dem Eingriff. In 3 weiteren Fällen wurde nur eine einmalige Blutung beobachtet, die nicht mit Sicherheit als Menstrualblutung zu bezeichnen ist.

In 12 Fällen sistirte die Periode nach der Keilresection vollständig. Ein Fall war bereits bei der Operation 5 Jahre nach der Menopause. Die anderen Fälle haben sich zum Theil nicht wieder eingefunden, zum Theil sind sie noch nicht lange genug beobachtet, um ein definitives Urtheil über das Verhalten der Menstruation zu gestatten. Dass sogar nach anscheinend vollständiger Entfernung der Corpus-Schleimhaut eine wohl aus der Cervix stammende Blutung mit menstruellem Typus eintreten kann beweist Fall 25.

Dass sich, wie'so häufig, auch in einigen unserer Fälle die chronische Metritis mit kleinen Myomen combinirte, die aber gegen das Bild der Metritis an Bedeutung zurücktraten, ist oben bereits erwähnt. Unter unseren 30 Metritisfällen ist $3 \mathrm{mal}$ ein kleines solitäres, 5 mal eine Anzahl von kleinen, theils intramuralen, theils subserösen Myomkeimen im Uterus gefunden worden.

Die Myome fielen meist in den zu resecirenden Keil; lagen sie in den seitlich übrig bleibenden Theilen des Uterus, so waren sie mit Leichtigkeit pon der Schnittfläche aus zu enucleiren. Uteri mit grösseren Myomen haben wir mit der Keilresection bisher nicht in Angriff genommen. Indessen dürfte es nicht von der Hand zu weisen sein, auch bei der vaginalen Operation grösserer Myome, wenn zugleich Prolaps besteht, seitlich beiderseits einen Rest des Uterus zur Bildung einer Pelotte zu erhalten. Nur muss man sicher sein, dass in den restirenden Theilen keine Myome enthalten sind.

Dass die Keilresection auch auf andere Erkrankungen des Uterus anwendbar ist, beweist Fall 21. Hier war die erhebliche Vergrösserung des Uterus bis auf Kindskopfgrösse auf eine chronische Metritis, vielleicht in Combination mit Myomen bezogen worden, da starke, die Patientin sehr schwächende Blutungen seit Jahren bestanden hatten. Bei der Operation zeigte sich, dass eine grosse pralle Cyste das Corpus uteri mit schleimhautähnlicher Auskleidung, die uns als ein bisher noch nicht beschriebenes Unicum erscheint ${ }^{1}$ ), zusammen mit einer beträchtlichen Verdickung der

1) Der Fall soll a. a. 0. ausführlich dargestellt werden. 
Uteruswand die Grösse der Gebärmutter bedingte. Da die Erhaltung einer Uteruspelotte für die Scheidenplastik wünschenswerth war und es sich um eine nicht maligne Bildung handelte, wurde die Keilresection des Corpus vorgenommen. Der grösste Theil des enormen Uteruskörpers wurde keilförmig resecirt, die seitlich stehenbleibenden Reste entsprachen an Grösse einer normalen Uterushälfte. Die glatte Cystenwand wurde vollständig entfernt. Die Musculatur der seitlichen Uterustheile zog sich sofort nach der Keilexcision zu einer dicken Lage zusammen, die an das Bild der Uteruswand nach dem Kaiserschnitt erinnerte. Durch Neoplastik des Uterus warde ein gutes plastisches Resultat erzielt. Die durchi die Keilresection von ihren Blutungen befreite, bis zur Operation sehr heruntergekommene Patientin stellte sich nach $1 / 4$ Jahr beschwerdefrei in sebr gehobenem Allgemeinzustand wieder vor.

In der Reconvalescenz haben wir nach der Keilresection des Corpus in einer grossen Anzahl der Fälle eine Störung zu verzeichnen, die uns als für die Keilresection beinahe charakteristisch erscheint. Es ist dies eine in den ersten Tagen der Convalescenz auftretende Temperatursteigerung, die uns bei unseren ersten Fällen etwas erschreckt hat, die wir aber bei weiteren Beobachtungen als ein harmloses Resorptionsfieber erkannt haben. Die Temperaturcurven, die an einem der ersten Tage post operationem steil in die Höhe geht, um schon am folgenden oder einem der nächsten Tage wieder abzusinken, entspricht ganz den Curven von Resorptionsfieber, wie wir sie z. B. nach der Operation tuberculöser Peritonitiden oder im Wochenbette beobachten. Der Puls und das Allgemeinbefinden bleiben dabei stets gut. Bakteriologische Untersuchungen des Wundsecrets zeigen keine Befunde virulenter Bakterien, und auch rein klinisch ist das Bild durchaus von dem der Infection verschieden. Derartige Resorptionsfieber haben wir nach Keilresection $13 \mathrm{mal}$, also fast in der Hälfte unserer Fälle beobachtet. Nur in 3 Fällen (1,4 und 26) musste eine leichte Infection ohne wesentliche Störung des Allgemeinbefindens für die Temperatursteigerung verantwortlich gemacht werden. Die übrigen 14 Fälle verliefen völlig glatt und ohne Temperatursteigerung.

Dass dieses Resorptionsfieber auf Rechnung der Keilresection zu setzen ist, geht daraus hervor, dass wir bei den zwischendurch in grosser Anzahl ausgeführten Vaginifixuren ohne Keilresection, die, wie oben angegeben, nach der gleichen Methode ausgeführt werden, derartige Temperatursteigerungen nicht beobachtet haben. 
Es muss also durch die breiten Schnittflächen bei der Keilresection, durch die Eröffnung und spätere Thrombosirung zahlreicher Gefässe, sowie durch die kleinen Nahtnekrosen im Corpus eine Summe todten Gewebes ausgeschaltet werden, die auf bisher noch unbekannte Art, wohl auf chemischem Wege, eine schnell ansteigende, aber auch bald wieder abfallende Temperaturerhöhung verursacht.

Eine Schädigung des Heilungsvorgangs oder eine Beeinträchtigung des Allgemeinbefindens haben wir durch dieses Resorptionsfieber in keinem Falle gesehen. Die Patientinnen wurden zum Theil schon in der Mitte, meist am Ende der 3. Woche geheilt und beschwerdefrei aus der Klinik entlassen. Nur bei einigen wenigen Frauen, die schon sehr anämisch und abgemagert die Klinik aufsuchten, haben wir zur Hebung des Allgemeinzustands eine etwas längere Anstaltspflege nöthig gehabt.

In fast allen unserer Fälle - einige liegen noch nicht lange genug zurück - haben wir nach mindestens 3 Monaten eine Nachuntersuchung vorgenommen, mehrere beobachten wir schon über ein Jahr nach der Operation. Stets haben wir ein gutes plastisches Operationsresultat und die Beseitigung der erschöpfenden und quälenden Metritis- und Prolapsbeschwerden constatiren können. Eine grosse Anzahl dieser Frauen, die vorher schon erfolglos durch verschiedene Hände gegangen waren, gehören zu unseren dankbarsten Patientinnen.

Wir hoffen, durch die Keilresection des Uteruskörpers eine erfolgreiche und schnell zum Ziele führende Behandlungsmethode für die Combination von chronischer Metritis und Prolaps angegeben zu haben. Eine solche Therapie ist insbesondere ein Bedürfniss für die Frauen der arbeitenden Klassen, denen wir keine langdauernde oder gar kostspielige conservative Therapie zumuthen können, sondern die wir möglichst schnell und definitiv von ihrem die Arbeitsfähigkeit beschränkenden oder sogar aufhebenden Leiden befreien müssen.

Im Anhang sind zur genaueren Erläuterung unserer Indicationsstellung und Technik, sowie der Operationsresultate die Krankengeschichten unserer Keilresectionen in möglichster Kürzung wiedergegeben.

Fall 1. 1903/636. Fr. L., 30 Jahre.

Anamnese: 3 spontane Geburten mit normalen. Wochenbetten. Seit der ersten Geburt vor 9 Jahren unregelmässig anteponirende, zeitweise sehr starke Periode. Seit einigen Jahren Senkungsbeschwerden. 
Bereits $2 \mathrm{mal}$ erfolglos ausgeschabt. In Jetzter Zeit schwere nervöse Beschwerden.

Status: Starker Descensus der vorderen und hinteren Vaginalwand. Grosser harter, anteflectirter, beweglicher, druckempfindlicher Uterus. Portio hypertrophisch.

Operation: In Aethernarkose quere vordere Kolpotomie, Abschieben der Blase. Spaltung der vorderen Cervixwand und Austastung des Uteruscavum, das sich leer erweist. Gründliche Abrasio. Hierauf Eröffnung des Peritoneum, Vorwälzen des stark vergrösserten Corpus. Die Wand ist verdickt und sehr brüchig. Resection eines medianen Keils aus dem Corpus mit Erhaltung von Corpus-Schleimbaut. Vernähung des Uterusrestes und vaginale Fixation desselben. Hintere Colporrhaphie und Dammplastik.

Reconvalescenz: Vom 2. bis 10. Tage Temperatursteigerung und viel Beschwerden. Höchste Temperatur 39,2 ${ }^{\circ}$ am 4. Tage. Am 11 ten Tage Abgang von Eiter per vaginam. Darauf Erleichterung und normale Temperatur.

Am 25. Tage geheilt entlassen.

Nachuntersuchung am 11. 10. 1904: Wesentliche Besserung. Nur zeitweise Ausfluss. Genitalbeschwerden sonst gering. Periode regelmässig, etwas schwach. Uterus klein, in guter Lage. Scheide eng. Zeitweise Darmblutungen und Schleim im Stuhl.

Fall 2. 1906/27. Fr. D., 47 Jahre.

Anamnese: 4 normale Geburten, letzte vor 7 Jahren. Seit 6 Jahren Senkungsbeschwerden.

Status: Totalprolaps. Massiges Collum. Ectropium. Dickes metritisches Corpus. Retroflexio mobilis.

Operation: In Aethernarkose vordere Kolporrhapbie, Keilresection aus der Mitte des Corpus. Einige intramurale Myomknötehen. UterusNeoplastik, vaginale Fixation des Uterus. Amputation beider Muttermundslippen. Kolporrhaphia posterior. Perineoplastik.

Reconvalescenz: Am 3. Tage nach der Operation Temperatursteigerung auf $38,3^{\circ}$. Sonst völlig glatte Reconvalescenz. Primäre Heilung. Höchste Pulsfrequenz 92. Am 18. Tage post oper. Entlassung. Gutes Operationsresultat.

Fall 3. 1906/111. Fr. V., 47 Jahre.

Anamnese: 9 normale Geburten und Wochenbetten, letzter Partus vor 3 Jahren.

Seit den letzten Geburten Senkungsbeschwerden, seit 3 Jahren unregelmässig anteponirende, sehr starke, 7-9 tägige Menstruation.

Status: Grosser metritischer beweglicher Uterus in Mittelstellung. Descensus uteri et vaginae mit Cystocele und Rectocele.

Operation: In Aethernarkose Fixurverfahren bis zum Hervorstürzen des Uterus. Aus dem Corpus wird ein grosser Keil mitsammt der Schleimhaut bis zum inneren Mutfermund excidirt. Vereinigung der restirenden Seitentheile des Uterus. Wegen der zahlreichen und starken Blutungen werden viele Omstechungen nöthig. Vaginale Fixation des Uterusrestes. Kolporrhaphia posterior. Perineoplastik.

Reconvalescenz: Vom 2. bis 6. Tage mässige Temperatursteigerung. Höchste Temperatur $38,6^{\circ}$ am 4. Tage. Höchste Pulsfrequenz 112. Sonst völlig reactionsloser Verlauf. Glatte Heilung. 
Nachuntersuchung nach 1/4 Jahr: Gutes plastisches Resultat. Wohlbefinden. Periode nicht wieder eingetreten.

Fall 4. 1906/146. Fr. H., 35 Jahre.

Anamnese: 3 spontane Geburten. Seit Jahren wegen Descensus Ringbehandlung. Nunmehr versagen die Ringe. Starke Blutungen. Druckschmerzen. Fluor.

Status: Starker Descensus. Grosses metritisches retroflectirtes Corpus.

Operation: Vordere Kolporrhaphie. Ablösung der Blase. Vorwälzen des Corpus., Keilförmige Resection des Corpus mit Ausschneiden der gesammteri Mucosa. Sehr blutreiche Operation. Neoplastik des Uterus. Vaginale Fixation des Stumpfes. Hintere Kolporrhaphie und ausgedehnte Dammplastik.

Reconvalescenz: In den ersten Tagen gestört, Temperatursteigerung bis $38,0^{\circ}$. Leichte peritonitische Reizung, Erbrechen, Singultus, Meteorismus. Am 5. Tage Rückgang der Erscheinungen, Hebung des Allgemeinbefindens. Am 6. Tage Nachmittags: Temperatur $38,3^{\circ}$, darauf Temperaturabfall und glatte Heilung.

Pat. verlässt am 13. Tage das Bett und wird am 22. Tage geheilt entlassen.

Nachuntersuchung nach 41/2 Monaten: Periode regelmässig, sehr schwach, ohne Beschwerden. Allgemeinbefinden gut. Plastisches Resultat vorzüglich.

Fall 5. 1906/217. Fr. Kl., 47 Jahre.

Anamnese: 9 normale Geburten und Wochenbetten, letzte vor 7 Jahren.

Seit über 1 Jahr starke Druckschmerzen im Unterleib und Senkungsbeschwerden. Regelmässige, nicht besonders starke Menses.

Status: Hochgradiger Prolaps der Vagina und des Uterus mit Cystocele und Rectocele. Uterus stark vergrössert, retroflectirt, beweglich.

Operation: In Aethernarkose vordere Kolporrhaphie. Hochschieben der Blase. Vorziehen des, faustgrossen Uterus. Keilexcision aus der Mitte des Uterus, wobei der untere Theil der Corpus-Schleimhaut erhalten bleibt. Die seitlichen Uterusstücke werden durch Knopfnähte wieder vereinigt, der neugebildete Uterus vaginal fixirt. Hintere Colporrhaphie und ausgiebige Dammplastik.

Reconvalescenz glatt. Normale Temperatur ausser einer einmaligen Steigerung auf $38,1^{\circ}$ am 5 . Tage.

Entlassung am 21. Tage mit normal grossem, gut gestrecktem Uterus und gutem Resultat der Scheidendammplastik.

$\mathrm{Nachuntersuchung}$ nach $/ / 4$ Jahr: Gutes Allgemeinbefinden. Vorzügliches plastisches Resultat. Vor 1 Monat 8 tägige, ziemlich starke "Periode".

Fall 6. 1906/290. Fr. V., 44 Jahre.

Anamnese: 5 Geburten. darunter ein Forceps, letzte vor 10 Jahren. Vor 8 Jahren wegen Gebärmuttervorfall operirt. Seit einiger Zeit wieder Senkungsbeschwerden; seit 1/2 Jahr Druckgefühl im Unterleib. Unregelmässige stark anteponirende Blutungen.

Status: Descensus vaginae, Grosser, beweglicher, retrovertirtflectirt liegender Utterus, 
Operation: Nach Abschieben der Blase Vorziehen des vergrösserten Uterus. Aus seiner Mitte wird ein grosses keilförmiges Stück excidirt, die Schleimhaut vollständig entfernt, Vereinigung der seitlichen Theilstïcke durch versenkte und oberflächliche Nähte. Vaginale Fixation des Uterusrestes. Hintere Kolporrhaphie und Dammplastik.

Reconvalescenz: Am 2. bis 4. Tage subfebrile Temperatur $\left(37,8^{\circ}\right)$. Am 5. Tage Temperatursteigerung auf $39,2^{\circ}$. Am 6. Tage definitiver Temperaturabfall. Efwas riechender bräunlicher Ausfluss, der durch Spülungen bald beseitigt wird. In der 3 . Woche kleine Thrombose am rechten Unterschenkel.

Entlassung am 22. Tage. Kleiner, gut gestreckt liegender Uterus. Gute Heilung der Scheidendammplastik.

Nachuntersuchung nach 11/4 Jahr: Wohlbefinden. Portio etwas hypertrophisch und etwas nach vorn sehend; sonst gutes plastisches Resultat. Periode nicht wieder eingetreten.

Fall 7. 1906/324. Fr. K., 33 Jahre.

Anamnese: 7 Geburten. Zahlreiche Druck- und Senkungsbeschwerden. Unregelmässige Blutungen, sehr stark, 9 tägig. Grünlich-schleimiger Fluor.

Status: Descensus der vorderen und hinteren Vaginalwand. Cystocele. Grosser harter metritischer Uterus.

Operation: In Aethernarkose vordere Kolpotomie. Abschieben der Blase. Mediane Spaltung der vorderen Cervixwand. Austastung des Cavum, das sich leer erweist. Nach Eröffnung des Peritoneum Hervorziehen des vergrösserten Uterus. Excision eines grossen Reils aus seiner Mitte, wobei der untere Theil der Corpus-Schleimhaut erhalten bleibt, um die Fortdaner der Menstruation zu ermöglichen. Vereinigung der beiden seitlichen Uterustheile. Schluss der vorderen Colpotomie. Hintere Kolporrhaphie und Dammplastik.

Reconvalescenz: Völlig glatt. Höchste Temperatur am 2. Tage $37,9^{\circ}$, sonst normale Temperatur.

Am 14. Tage geheilt und beschwerdefrei entlassen.

Nachuntersuchung nach 13 Monaten: Periode regelmässig, dreitägig, schwach, obne Beschwerden. Allgemeinbefund gut. Plastisches Resultat gut.

Fall 8. 1906/326. Fr. D., 36 Jahre.

Anamnese: 6 Geburten, letzte vor 7 Jahren. Mehrere Wochenbetten fieberhaft.

Sei 10 Jahren starke, unregelmässig anteponirende Periode. Deswegen zweimal Abrasio mit nur vorübergehendem Erfolge. Seit ca. 1 Jahr bedeutende Verschlimmerung der Beschwerden. In Zwischenräumen von 14. Tagen 8 Tage dauernde starke Blutungen. Heftige Schmerzen im Rücken, besonders in der Kreuzgegend, die unabhängig von der Blutung auftreten und Patientin arbeitsunfähig machen.

Status: Mässiger Descensus vaginae. Ueber faustgrosser, anteflectirter, beweglicher harter Uterus.

Operation: In Aethernarkose vordere Kolporrhaphie. Abschieben der Blase. Vorziehen des über faustgrossen Uterus. Aus der Mitte des Uterus wird ein grosser Keil excidirt, dessen Basis im Fundus, dessen Spitze im oberen Theil der Cervix liegt. Die gesammte Corpusschleimhaut ist in dem Keil enthalten. Ziemlich starke Blutung aus zahlreichen Gefüssen. Vernähung der beiden seitliehen Uterushälften durch tief- 
greifende und oberflächliche Nähte. Vaginale Fixation des Uterusrestes. Scheidendammplastik.

Reconvalescenz gestört. Vom 2. bis 9. Tage Temperatursteigerung. Höchste Temperatur am 3. Tage $39,0^{\circ}$. Höchste Pulsfrequenz 116. Thrombose im linken Oberschenkel, die nach der Inguinalbeage fortschreitet, auf Hochlagerung und essigsaure Tonerde-Umschläge zurückgeht. Vom 10. Tage an normale Temperatur.

Entlassung am 45. Tage. Uterus von der Grösse eines normalen Organs und von weicher Consistenz, gut gestreckt. Scheide eng. Völlig beschwerdefrei.

Nachuntersuchung nach 1/4 Jahr: Wohlbefinden. Tadelloses plastisches Resultat. Uterus klein, gut gestreckt. Periode nicht wieder eingetreten.

Fall 9. 1906/376. Fr. Kl., 40 Jahre.

Anamnese: 4 Geburten ohne Complicationen, letzte vor 4 Jahren.

Seit 6 Jahren starke, in letzter Zeit sehr entkräftende Periode von 8 tägiger Dauer mit nur 14 tägigen Pausen. Seit einem Jahre Ab. magerung und starkes Schwächegefühl, so dass Pat. arbeitsunfähig ist. Starker gelblicher Fluor.

Status: Erbeblicher Descensus vaginae.

Der Uterus liegt retrovertirtflectirt, lässt sich leicht aufrichten. Corpus über faustgross, hart.

Operation: In Aethernarkose Probe-Abrasio, die hyperplastische Schleimhaut ergiebt. Vordere Kolporrhaphie. Hochschieben der Blase. Vorleiten des über faustgrössen metritischen Corpus. Resection eines breiten Keils, welcher die gesammte Mucosa einschliesst. Es blutet stark. Seitliche Tubenunterbindung schaltet beiderseits die Anastomose mit der Spermatica aus. Reconstruction des Corpus-Restes, der vaginal fixirt wird. Ausgiebige hintere Kolporrhaphie, hohe Perineoplastik.

Reconvalescenz glatt. Höchste Temperatur 38,0 .

Entlassung am 21. Tage, Kräftezustand bedeutend gehoben. Uterus normal gross, gut gestreckt. Gutes Resultat der Scheidendammplastik.

Nachuntersuchung nach $1 / 4$ Jahr: Wohlbefinden, gutes plastisches Resultat. Periode nicht wieder eingetreten.

Fall 10. 1906,579. Fr. F., 42 Jahre.

Anamnese: 6 Geburten, darunter ein Forceps, letzte vor 3 Jahren. Wochenbetten afebril.

Seit 5 Jahren Senkungsbeschwerden und Heraustreten der Scheide, seit der letzten Geburt verschlimmert. Ringbehandlung erfolglos.

Menses regelmässig, nicht besonders stark. Fluor.

Status: Uterus stark vergrössert, in Mittelstellung, sehr beweglich. Rechtsseitiger alter Cervixriss.

Ectropium der Cervicalschleimbaut. Portio wulstig.

Descensus der vorderen und hinteren Vaginalwand. Rectocele.

Operation: In Aethernarkose zuerst Portioamputation. Dann ausgiebige vordere Kolporrhaphie. Abschieben der Blase. Hervorziehen des sehr grossen Uterus. Excision eines grossen Keils mit vollkommener Ausschneidung der Corpus-Schleimhaut. Vereinigung der übrig bleibenden Uterusseiten durch versenkte und oberflächliche Nähte. Vaginale Fixation des Uterus. Hintere Kolporrhaphie. Perineoplastik.

Reconvalescenz glatt. Höchste Temperatur 38,0 am 6. Tage. 
Am 21. Tage mit gutem Operationsresultat und subjectivem Wohlbefinden geheilt entlassen.

Nachuntersuchung nach 1/4 Jahr: Vorzügliches plastisches Resultat. Wohlbefinden. Einmal schwache 2 tägige Periode.

Fall 11. 1906/601. Fr. N., 35 Jahre.

Anamnese: Multipara. Letzte Geburt vor 10 Jahren. Geburt.

Starke Druckbeschwerden. Vortreten der Scheide seit der letzten

Status: Starker Descensus der vorderen und hinteren Vaginalwand. Alter Dammriss. Grosser harter Uterus, dextrovertirt, hinten fixirt, leicht retroflectirt: Am Fundus kleines subseröses Nyom. Bräunlicher Fluor.

Operation: In Aethernarkose Kolpotomie, Hochschieben der Blase. Vorziehen des dicken grossen Uterus. Resection des Corpus-Mittelstücks mit dem haselnussgrossen subserösen Myom. Ein im excidirten Keil nicht enthaltener zurückgebliebener Rest der Corpus-Schleimhaut wird mit scharfem Schablöffel abgeschabt. Restituirung des Corpus-Restes. Vaginale Fixation des nenen Uterus. Kolporrhaphia posterior. Perineoplastik.

Reconvalescenz völlig glatt. Normale Temperatur.

Am 18. Tage geheilt und beschwerdefrei entlassen.

Nachuntersuchung nach 1 Jahr: Wohlbefinden. Gutes plastisches Resultat. Blutung nur eiumal schwach wieder eingetreten.

Fall 12. 1906/640. Fr. St., 32 Jahre.

Anamnese: 6 Geburten, darunter ein Forceps, bei den letzten 3 Geburten starke Blutung. Wochenbetten fieberfrei, Letzte Geburt vor 3 Jahren.

Unregelmässig anteponirende Menses.

Vor 7 Jahren wegen Scheidensenkung Scheidendammplastik. Seit mehreren Jahren wieder Scheidenvorfall, Druckbeschwerden nach unten und auf die Blase.

Status: Descensus der vorderen und hinteren Vaginalwand. Cystocele. Rectocele. Uterus vergrössert, hart, anteflectirt, beweglich.

Operation: In Aethernarkose vordere Kolporrhaphie. Ablösen der Blase. Vorziehen des stark vergrösserten Uterus. Provisorisches Abklemmen der Ligamente. Excision eines grossen medianen Keils aus dem Corpus mit Entfernung der gesammten Corpus-Schleimhaut. Vernähung der beiden seitlichen Stümpfe, vaginale Fixation des neugebildeten Uterus. Ausgiebige hintere Kolporrhaphie und Dammplastik.

Reconvalescenz: In der ersten Woche leichte Temperatursteigerungen. Höchste Temperatur $38,3^{\circ}$ am 5. Tage. Sonst glatte Reconvalescenz und gute Heilung.

Entlassung am 25. Tage. Uterus normal gross, gut gestreckt. Gutes Resultat der Scheidendammplastik. Wohlbefinden.

Nachuntersuchung nach $1 / 4 \mathrm{Jahr}$ : Gutes plastisches Resultat. Ausser einigen nervösen Beschwerden Wohlbefinden. Periode nicht wieder eingetreten.

Fall 13. 1906/657. Fr. Pf., 56 Jahre.

Anamnese: 2 spontane Geburten mit fieberfreiem Wochenbett, letzte vor 23 Jahren. Seit 5 Jahren Mepopause. Seit 8 Jahren Scheidenvorfall, deshalb Ringbehandlung, die jetzt nicht mehr ausreicht. In 
Status: Systolisches Geräusch an der Herzspitze.

Hochgradige Inversion beider Vaginalwände mit Rectocele und grosser Cystocele. Uterus anteflectirt, beweglich, etwas vergrössert, mit mehreren kleinen subperitonealen Myomknollen an der Vorder- und Hinterwand.

Operation: In Aethernarkose vordere Kolporrhaphie. Hochschieben der Blase. Vorziehen des mit kleinen Myomen besetzten Uterus. Provisorische Abklemmung der Ligamente. Der grösste Theil des Corpus wird median keilförmig excidirt. Die kleineren seitlichen Stümpfe werden vereinigt. Die so gebildete Pelotte wird vaginal fixirt. Hintere Colporrhaphie und Dammplastik.

Reconvalescenz: Am 2. und 3. Tage Temperatursteigerung, höchste Temperatur $38,5^{\circ}$. Bls zum 6. Tage subfebrile Temperatur, dann normale Temperatur. Bis zum 15. Tage Unmöglichkeit, spontan Urin $\mathrm{zu}$ lassen.

Entlassung am 24. Tage. Kleiner gut gestreckter Uterus. Gutes plastisches Resultat. Wohlbefinden.

Nachuntersuchung nach 1/2 Jahr: Gutes Operationsresultat. Keine Beschwerden.

Fall 14. 1906/661. Fr. Gr., 43 Jahre.

Anamnese: 2 Geburten, mit Forceps beendet, letzte vor 7 Jahren. Im ersten Wochenbett Fieber.

2 Aborte im 2. Monat.

In den letzten Jahren sehr starke, etwas anteponirende, die Patientin sehr schwächende Menses. Druckgefühl im Unterleib und im Rücken, Schmerzen bei der Cohabitation. Reichlicher Fluor. Abrasio und Amputation der hypertrophischen Portio, in biesiger Klinik ausgeführt, ohne Erfolg. Die Blutungen sind seitdem noch stärker geworden. Patientin wünscht jetzt dringend definitive Beseitigung des Leidens.

Status: Geringer Descensus vaginae. Uterus auch ohne die amputirte Portio noch vergrössert, in Mittelstellung, beweglich.

Operation: In Aethernarkose vordere Kolporrhaphie und Hochschieben der Blase. Wegen Verdachts auf intrauterinen Polypen zunächst vorderer Eröffnungsschnitt und Austastung des Cavum. Dieses erweist sich als leer. Vorwälzen des metritisch verdickten Uteruskörpers. Excision eines Keils aus dem Corpus mit Schonung des unteren Theils der Corpus-Schleimhant, um nach Wunsch der Patientin die Menstruation zu erhalten. In der Cervix findet sich ein kaum haselnussgrosses intramurales Myom, das enucleirt wird. Vereinigung der beiden seitlichen Uterusstümpfe und Verschluss der Cervix mit Bildung eines kleinen, von Schleimhaut ausgekleideten Uterus-Cavum. Vaginale Fixation des Uterusstumpfs. Ziemlich blutige Operation.

Reconvalescenz völlig glatt. Keine Temperatursteigerung. Zu dem erwarteten Termin tritt die Menstruation nicht ein.

Entlassung am 25. Tage. Wohlbefinden. Der kleine Uterusstumpf liegt gut fixirt.

Laut brieflicher Mittheilung der nach Amerika verzogenenen Patientin ist die Periode nicht wieder eingetreten. Patientin ist jetzt vollkommen beschwerdefrei.

F all 15. 1906/666. Fr. Kü., 33 Jahre.

Anamnese: 1 Geburt vor $11 / 2$ Jahren, spontan mit fieberfreiem Wrohenheti. Seitdem starke Druckbeschwerden und Scheidensenkung. 
Unregelmässig anteponirende starke Blutungen. Bei verschiedenen Gynäkologen in Behandlung; ohne Erfolg.

Status: Descensus des Uterus und der vorderen Vaginalwand. Hyperplastisches Collum, schnabelförmige Verlängerung der vorderen Muttermundslippe. Uterus faustgross, antefiectirt, beweglich, von harter Consistenz. Von der hinteren Uteruswand hängt ein hühnereigrosses Nyom beweglich in den Douglas. Ovarien etwas gross, aber frei beweglich.

Operation: In Aetheruarkose Excision beider hypertrophischer Muttermundslippen. Vordere Kolporrhaphie, Hochschieben der Blase. Eröffnung des Peritoneum. Stürzen des mächtigen Uterus. Abtragen des langgestielten subserösen Myoms der Hinterwand. Ausgiebige Keilresection des Corpus, der Keil enthält einige kleine intramurale Myomkeime, die Mucosa der Tubenecken wird resecirt. Der untere Theil der Corpus-Schleimhaut wird zur Erhaltung der Menstruation geschont. Neoplastik des Uterus, der vaginal fixirt wird.

Reconvalescenz völlig glatt. Keine Temperatursteigerung. Pat. verlässt am 9. Tage das Bett, wird am 18. Tage geheilt und beschwerdefrei entlassen. Die Menses sind zunächst am erwarteten Termin nicht eingetreten.

Nachuntersuchung nach $3 / 4$ Jahr: Menses regelmässig, sehr schwach, obne Beschwerden. Plastisches Resultat gut. Keine localen Beschwerden.

Fall 16. 1906/725. Fr. Kö., 42 Jahre.

Anamnese: 3 spontane Geburten mit normalen Wochenbetten. In den letzten Jahren sehr starke, unregelmässig anteponirende Blutungen. Heftige Druckbeschwerden. Vortreten der Scheide.

Status: Descensus beider Vaginalwände mit grosser Cystocele. Niedriger Damm. Uteruskörper faustgross. Retroflexio uteri. Der Uterus ist hinten fixirt, der Douglas ist verlötet und sehr druckempfindlich. Linksseitiger eigrosser Adnextumor, auf Druck schmerzhaft. Rechtsseitige Schenkelhernie.

Operation: In Aethernarkose zuerst Radicaloperation der Schenkelhernie. Darauf vordere Kolporrhaphie, Hochschieben der Blase, Eröfnung des Peritoneum. Der Uterus ist allseitig verwachsen, wird gelöst. Der linksseitige hühnereigrosse Tuboovarialtumor wird entfernt. Keilresection aus dem Corpus uteri. Der Keil enthält ein kirscbgrosses subseröses Myom. Neoplastik des Uterus and vaginale Fixation desselben. Das vordere Peritoneum wird über dem Rücken des Fundus aufgenäht. Ausgiebige hochreichende hintere Kolporrhaphie und Perineoplastik. Starke Verengerung der Vulva und Vagina.

Reconvalescenz: In der ersten Woche leichte Temperatursteigerungen. Höchste Temperatur 38,2 am 6. Tage. Sonst glatte Reconvalescenz. Schöne prima intentio.

Am 22. Tage geheilt und beschwerdefrei entlassen.

Nachunters uchung nach 10 Mon.: Woblbefinden. Kein Druckgefühl mehr. Periode regelmässig, schwach, 6- -7tägig, ohne Schmerzen. Beim Gehen etwas Ausfluss. Gutes plastisches Resultat,

Fall 17. 1906/737. Fr. B., 39 Jahre.

Anamnese: Mebrere spontane Geburten. Seit 3 Jahren wegen Scheidenvorfall mit Ring behandelt. Lnregelmässig anteponirende starke Periode. 
Status: Hochgradiger Descensus mit Cystocele. Grosses metritisches Corpus, an der Hinterwand fixirt, schnappt nach dem Aufrichten sogleich wieder zurück. Portio stark hypertrophisch. BasedowSymptome,

Operation: In Aethexnarkose vordere Kolporrhaphie. Hochschieben der Blase. Dann hohe Amputatio portionis. Darauf Eröffnung des Peritoneum, Vorleiten des mächtig verdickten Corpus uteri. Keilresection aus dem Corpus mit Entfernung "der gesammten Corpusschleimhaut. Wiedervereinigung der seitlichen Uterusreste. Vaginale Fixation des neuen Uterus. Hochreichende, sehr ausgiebige hintere Kolporrhaphie und Perineoplastik.

Reconvalescenz röllig glatt; höchste Temperatur $37,9^{\circ}$ am 2. Tage, sonst ganz normale Temperatur. Pat. verlässt am 11. Tage das Bett und wird am 15. Tage geheilt und beschwerdefrei entlassen. Tadellose Heilung der Plastik.

Nachuntersuchung nach 1/2 Jahre. Keine Beschwerden. Vorzügliches plastisches Resultat. Periode nicht wieder eingetreten.

Fall 18. 1906/757. Fr. Si, 40 Jahre.

Anamnese: 5 spontane Geburten, letzte vor 5 Jahren. Fast in allen Wochenbetten Fieber mit Schüttelfrost. Seit ca. 1 Jahre unregelmässig anteponirende Periode mit intramenstruellen Schmerzen. Ebenfalls seit einem Jahre Druckgefühl nach unten und auf die Blase. Häufiger Urindrang. Vortreten der Scheide. Starker weisslicher Fluor. Abmagerung, Schwächegefühl, Appetitlosigkeit.

Status: Klaffender Introitus. Starker Descensus beider Vaginalwände mit Cystocele und Rectocele. Uterus in beweglicher, leicht aufrichtbarer Retroversio-flexio. Corpus stark vergrössert, hart. Portio and Collum hypertrophisch.

Operation: In Aethernarkose zunächst Probe-Abrasio, die gutartige Schleimhautbröckel in geringer Menge zu Tage fördert, Sondenlänge $14 \mathrm{~cm}$. Ausgiebige Amputation der hypertrophischen Portio. Vordere Kolporrhaphie. Hochschieben der Blase. Vorleiten des grossen Corpus, aus dem ein grosser Keil mit der gesammten Corpusschleimbaut resecirt wird. Vereinigung der seitlichen Uterusreste und vaginale Fixation des neugebildeten Organs.

Reconvalescenz: Am Nachmittage des 2. Tages Temperatursteigerung auf $38,9^{\circ}$, am Vormittage des 3. Tages auf 39,0. Dabei ungestörtes Allgemeinbefinden. Am Nachmittage des 3. Tages Temperaturabfall, vom 5 Tage ab völlig normale Temperatur und glatte Reconvalescenz.

Am 22. Tage mit subjectivem Wohlbefinden und vorzüglichem plastischen Resultat entlassen. Uterus normal gross, gut gestreckt.

Nachuntersuchung nach $1 / 4$ Jahr: Gutes Operationsresultat. Ausser leichtem Druckgefühl keine Beschwerden. Periode nicht mehr eingetreten.

Fall 19. 1906/795. Fr. M., 36 Jahre.

Anamnese: Pat. ist steril verheiratet. Seit Jahren sehr starke, unregelmässig anteponirende Menorrhagien mit dysmenorrhoisehen Beschwerden. Heftige Druckbeschwerden im Leib, besonders auf die Blasengegend. Seit Jahren ohne Erfolg in gynäkologischer Behandlung.

Status: Mässicer Descensus der vorderen Vaginalwand, kleine 
Cystocele. Corpus leicht anteflectirt, hart, stark vergrössert. In der vorderen Cervixwand ein walnussgrosses Myom.

Operation: In Aethernarkose vordere Kolpotomie, $\mathbb{1}$-Schnitt. Vorleiten des stark vergrösserten Uterus, welchem etwas serös blutiger Ascites folgt. Das Corpus ist schwer metritisch verändert. Ausserdem findet sich in jedem Tubenwinkel ein kleiner Myomknollen, sowie im linken Horn an der Hinterfläche eine glasige Cyste mit Nebencystchen. Daher wird eine ausgedehnte Keilresection des Corpus vorgenommen, die ausser den Cysten und den Tubenwinkelknollen die ganze Corpusmucosa entfernt. Nach der Resection wird der tief im unteren Uterinsegment sitzende walnussgrosse Myomknoten enucleirt. Zum Schluss Uterus-Neoplastik und vaginale Fixation des Corpusrestes. Quere Vereinigung der 1 -förmigen Kolpotomiewunde.

Reconvalescenz äusserst glatt. Keine Temperatursteigerung. Primäre Wundheilung. Patientin ist psychisch äusserst günstig beeinflusst, verlässt am 10. Tage das Bett und wird am 17. Tage geheilt mit gutem plastischen Resultat entlassen.

$\mathrm{Nachuntersuchung}$ nach $1 / 4$ Jahr. Dysmenorrhoe versehwunden, ebenso die Schmerzen im Leib. Allgemeinbefinden gut. Periode regelmässig, schwach. Plastisches Resultat gut. Uterus sehr klein.

Fall 20. 1907/119. Fr. F. 46 Jahre.

Anamnese: 3 spontane Geburten mit normalen Wochenbetten. Seit vielen Jahren sehr starke, unregelmässig anteponirende Blutungen. Heftiges Druckgefïhl im Leib. Starkes Vortreten der Scheide.

Status: Hochgradiger Descensus der vorderen und hinteren Scheidenwand. Grosse Cystocele. Damm schlaff und niedrig. Selir grosser harter Uterus, in Mittelstellung. Collum breit und hart. Eetropium beider Muttermundslippen.

Operation: In Aetheruarkose Amputation beider Huttermundslippen. Vordere Kolporrhaphie. Auslösung und Hochschieben der Blase. Vorleiten des stark verdickten Uteruskörpers. Resection eines breiten Mittelkeils aus dem Corpus. Enucleation mehrerer kleiner, theils intramuraler, theils subseröser Myomkeime von den Schnittflächen aus. Die gesammte Corpusmucosa wird entfernt. Bildung eines neuen schmalen Uterus von ziemlicher Länge und vaginale Fixation desselben. Ausgiebige hintere Kolporrhaphie und Perineoplastik.

Reconvalescenz glatt. Keine Temperatursteigerung. Primäre Wundheilung. Pat. verlässt am 12. Tage das Bett und wird am 22. Tage beschwerdefrei und geheilt entlassen.

Nachuntersuchung nach 4 Mon. Guter localer Befund. Ausser Cystitisbeschwerden Wohlbefinden. Menses nicht wieder eingetreten.

Fall 21. 1907/133. Fr. H. 42 Jahre.

Anamnese: 4 spontane Geburten, im 2. Wochenbett Fieber. Seit 10 Jahren starke aber regelmässige Periode. Seit der ersten Geburt Scheidensenkung. Seit der letzten Geburt vor 2 Jahren bedeutende Verschlimmerung des Vorfalls, reichlicher Fluor. Heftiges Druckgefübl im Leib, besonders auf die Bláse. Rückeuschmerzen. Pat. ist jetzt durch die starken Beschwerden arbeitsunfähig.

Status: Nagere anämische Frau. Schlaffe Bauchdecken. Introitus klaffend, alter Dammriss. Scheide weit. Starker Descensus der vorderen und hinteren Vaginalwand. Cystocele. Portio stark hyperplastisch mit beiderseitigen Einrisseu. Uteruskörper stark vergrössert, dextrovertirt 
und retroponirt, lïsst sich uach vorn bringen, scheint aber am unteren Theil der Hinterwand fixirt zu sein.

Operation: In Aethernarkose Amputation beider ektropionirter Muttermundslippen. Vordere Kolporrhaphie. Hochschieben der Blase. Vorleiten des stark metritisch verdickten Corpus. Dabei reissen an der Hinterfläche des Uterus sehr feste alte Douglasadhäsionen zwischen Appendices epiploicae, beiden Adnexen und Cervix-Corpus-Grenze ein. Keilresection des Corpus mit Entfernung der ganzen Mucosafläche. Vereinigung der seiflichen Uterustheile, vaginale Fixation des neuen Corpus. Kolporrhaphia posterior und Perineoplastik.

Reconvalescenz völlig glatt. Keine Temperatursteigerung. Primäre Wundheilung. Pat. steht am 12. Tage auf und wird am 20. Tago entlassen. Der neue Uterus ist von normaler Grösse und gut gestreckt. Gutes Resultat der Scheidendammplastik.

Nachuntersuchung nach $1 / 4$ Jahr. Pat. fühlt sich noch etwas schwach, bat sonst keine Beschwerden mehr. Gutes plastisches Resultat. Menses nicht wieder eingetreten. Allgemeinzustand sehr gehoben.

Fall 22. 1907/171. Fr. Kr. 38 Jahre.

Anamnese: 3 Geburten, darunter ein Forceps, letate vor zehn Jahren. Im ersten Wochenbett Fieber. Vor 9 Jahren wegen Scheidensenkung Seheidendammplastik. Soit 6 Jahren sehr starke unregelmässig anteponirende, schwächende und mit beftigen intramenstruellen Schmerzen verbundene Periode. Ausserhalb der Periode lebhafte Druckschmerzen im Leib, Beschwerden beim Wasserlassen, Kreuzschmerzen. Letzte Periode vor 3 Wochen. Keine Cessatio.

Status: Weiter Introitus, mässiger Descensus der vorderen Scheidenwand, kleine Cystocele. Uterus kleinkindskopfgross, bis 3 Querfinger unterhalb des Nabels reichend, anteflectirt, beweglich. Die Consistenz des Uterus ist von auffallender cystisch-weicher Beschaffenheit. Durch längere Beobachtung wird beim Fehlen aller Schwangerschaftssymptome das Bestehen einer Gravidität mit Sicherheit ausgeschlossen. Da Pat. auf Beseitigung ihrer heftigen Beschwerden dringt, wird in Aethernarkose die Operation vorgenommen.

Operation: Vordere Kolporrhaphie. Hochschieben der Blase. Spaltung der vorderen Cervixwand zur Austastung. Bei Eröffnung des inneren Muttermundes entleert sich plötzlich gelbliche klare Flüssigkeit in reichlicher Menge (schätzungsweise $300 \mathrm{ccm}$ ). Die Austastung des Cavum ergiebt, dass dasselbe leer ist und keinerlei Schwangerschaftsproducte enthält. Die Auskleidung der Uterusböhle bildet eine glatte Cystenwand, die sich nur mit grosser Gewalt in kleinen Fetzen abziehen tässt. ") Nach Eröffnung der vorderen Plica peritonei wird der Uterus vorgezogen, der einen noch immer sehr grossen schlaffen Sack mit dicker Wandung darstellt. $\mathrm{Da}$ die Erhaltung einer Uteruspelotte für die Seheidenplastik wünschenswerth ist und es sich nicht um eine maligne Neubildung handelt, wird nicht die Totalexstirpation, sondern die Keilresection des Uterus vorgenommen. Der grösste Theil des. enormen Corpus wird keilförmig resecirt. Die seitlichen Reste entsprechen an Grösse einer normalen Uterushälfte. Die Cystenwand wird

1) Mikroskopisch zeigte die Cystenwand einen schleimhautähnlichen Charakter von schwer zu deutender Herkunft. Ein Analogon dafür haben wir in der Literatur nicht auffinden können. 
vollständig entfernt. Die Muskulatur der seitlichen Uterustheile zieht sich sofort zu einer dicken Lage zusammen, die an das Bild der Uteruswand nach dem Kaiserschnitt erimnert. Neoplastik des Uterus durch tiefgreifende Knopfnähte. Vaginale Fixation des neugebildeten Organs. Vermähung des Cervixschnittes und der Kolporrhaphie.

Reconvalescenz: Am 2. Tage Temperatursteigerung auf $38,6^{\circ}$. Darauf subfebrile Temperaturen bis zur Mitte der 2. Woche bei gutem Allgemeinbefinden. Keine Secretion. Patientin verlässt am 17. Tage das Bett und wird am 22. Tage beschwerdefrei mit guter Heilung entlassen. Der normal grosse Uterus liegt gut gestreckt.

Nachuntersuchung nach $1 / 4$ Jahr. Subjectiv Wohlbefinden. Objectiv gutes Operationsresultat mit normal grossem, gestreckt liegendem Uterus. Menses nicht wieder eingetreten.

Fall 23. 1907/190. Fr. N., 49 .Jahre.

Anamnese: 5 spontane Geburten, letate vor 8 Jahren. In mehreren Wochenbetten Fieber. Seit 4 Jahren starke unregelmässig anteponirende Blutungen. Deshalb vor 2 Jahren in hiesiger Klinik Austastung des Uterus, der leer gefunden wurde, und Abrasio. Dieselbe blieb ohne Erfolg. Durch die Blutungen ist Patientin in letzter Zeit sehr geschwächt. Ausserdem starke Druckschmerzen im Leib und Vortreten der Scheide. Patientin wünscht dringend definitive Beseitigung des Zustands, der sie arbeitsunfähig macht.

Status: Ziemlich anämische Frau. Keine nachweisbaren Herzveränderungen. Introitus klaffend, alter Dammriss. Starker Descensus der vorderen und hinteren Vaginalwand mit Cystocele und Rectocele. Uterus stark metritisch vergrössert, hart, anteflectirt, beweglich.

Operation: In Aethernarkose breite vordere Kolporrhaphie, Hochschieben der Blase, Hervorholen des stark verdickten und sehr harten Corpus. Excision eines keilförmigen Stückes aus dem Uteruskörper mit Entfernung der gesammten Uterus-Schleimhaut. Vernähung der übrig gebliebenen seitlichen Uterustheile. Vaginale Fixation des neugebildeten Uterus. Hintere Kolporrhaphie und Dammplastik.

Reconvalescenz: Am 3. und 4. Tage Temperatursteigerung bis $38,9^{\circ}$ bei gutem Allgemeinbefinden. Puls 124. Vom 6. Tage an wieder normale Temperatur und glatte Reconvalescenz. Geringer, etwas riechender Fluor. Gute Wundheilung. Vom 11.-13. Tage nach Autsetzen im Bett wieder etwas Temperatursteigerung. Am 15. Tage verlässt Pat. das Bett und wird am 20. Tage geheilt und beschwerdefrei entlassen. Der Uterus ist normal gross und gut gestreckt; gutes Resultat der Scheidendammplastik.

Nachuntersuchung nach 1/4 Jahr: Wohlbefinden. Gutes plastisches Resultat. Keine Blutung mehr.

Fall 24. 1907/306. Fr. A., 43 Jahre.

An amnese: 10 spontane Geburten, letzte Zwillinge, vor 3 Jahren. Wochenbetten afebril. Seit vielen Jahren mit starkem Blutverlust vorbundene, aber regelmässige Periode. Seit der letzten Geburt heftige Druckschmerzen im Leib und Vortreten der Scheide. Vom Arzt ohne Erfolg mit Ring behandelt.

Status: Introitus klaffend. Alter Dammriss. Starker Descensus der vorderen und hinteren Vaginalwand mit geringer Cystocele und Rectocele. Uterus metritisch vergrössert, bart, retroflectirt, unbeweglich nach hinten fixirt. 
Operation: In Medullaranästhesie vordere Kolporrhaphie. Hochschieben der Blase. Nach Eröffnung des Peritoneum langsames Aufrichten und Lösen des Uterus aus den ihn nach hinten fixirenden perimetritischen Adhäsionen. Das vorgezogene Corpus ist metritisch vergrössert. Keilresection aus dem Fundus, wobei der obere Theil der Corpus-Scbleimhaut zur Erhaltung der Menstruation erhalten bleibt. Neuformirung des Uterus aus den seitlichen Stücken und vaginale Fixation desselben. Hintere Kolporrhaphie und Perineoplastik.

Reconvalescenz: Völlig glatte, primäre Heilung. Keine Temperatursteigerung. Pat. steht am 12. Tage auf und wird am 21. Tage beschwerdefrei entlassen. Der neue Uterus ist normal gross und gut gestreckt; gutes Resultat der Scheidendammplastik.

Nachuntersuchung nach $1 / 4 \mathrm{Jahr}$ : Seit der Operation 2 maliges Auftreten einer normal starken, 4 Tage dauernden Menstruation, ohne Beschwerden. Völliges Wohlbefinden. Uterus von normaler Grösse, gut gestreckt. Introitus und Scheide eng. Damm hoch und fest.

Fall 25. 1907/319. Fr. H., 39 Jahre.

Anamnese: 7 spontane Geburten, im ersten Wochenbett Fieber. Bei der 4. Geburt manuelle Placentarlösung. Seit mehreren Jahren mit starkem Blutverlust verbundene, regelmässig anteponirende, in letzter Zeit meist 8 Tage dauernde Periode. Durch die Blutungen ist Pat. jetzt sehr geschwăcht und arbeitsunfähig. Seit den letzten Geburten Vorfall der Scheide.

Status: Ziemlich anämische Frau. Systolisches Geräusch an der Herzspitze. Herzgrenzen nicht verbreitert. Klaffender Introitus. Starker Descensus der vorderen und hinteren Vaginalwand. Cystocele und Reetocele. Uterus stark metritisch vergrössert, anteflectirt, beweglich.

Operation: In Medullaranästhesie vordere Kolporrhaphie, Hochschieben der Blase. Vorziehen des metritischen faustgrossen Uteruskörpers. Beiderseitige Unterbindung der Tube zur Ausschaltung der von der Spermatica kommenden Anastomosen. Mediale Excision eines grossen Keils aus dem Corpus und Entfermung der ganzen CorpusSchleimhaut. Mässige Blutung. Vereinigung der Seitentheile zu einem neuen Uterus, auf dessen Hinterfläche das vordere Peritoneum aufgenäht wird. Vaginale Fixation des neuen Uterus. Hintere Kolporrhaphie und Dammplastik.

Reconvalescenz: Am Nachmittage des Operationstages $38,4^{\circ}$, am 2. Tage $38,0^{\circ}$. Darauf normale Temperatur. Stets gutes Allgemeinbefinden. Primäre Wundheilung. Pat. steht am 10. Tage auf, wird am 18. Tage beschwerdefrei entlassen. Das Resultat der Scheidendammplastik ist vorzüglich, der neue Uterus ist von normaler Grösse und liegt gut gestreckt.

Nachuntersuchung nach $1 / 4 \mathrm{Jahr}$ : $5^{1 / 2}$ Wochen nach der Operation Auftreten einer schwachen, 2 Tage dauernden Menstrualblutung. 4 Wochen darauf normal starke, 5tägige Periode, beschwerdefrei. Völliges Wohlbefinden. Gutes plastisches Resultat. Uterus normal gross.

Fall 26. 1907/383. Fr. L., 45 Jahre.

Anamnese: 5 spontane Geburten, letzte vor 10 Jahren. Wochenbetten fieberfrei. Periode seit der letzten Geburt viel stärker als früher, 8 Tage dauernd, unregelmässig anteponirend mit prä- und intramenstruellen Schmerzen. Im letzten Jahre ist die Periode mit sehr starkem, schwächendem Blutverlust verbunden und dauert meist 14 Tage 
an. Starkes Druckgefühl im Leib. Senkung der Scheide, deshalb Ringbehandlung, ohne viel Erfolg.

Status: Sehr ausgeblutete, magere Frau. Keine Herzveränderungen nachweisbar. Introitus klaffend, alter Dammriss. Hochgradiger Descensus der vorderen und hinteren Vaginalwand mit Cystocele und Rectocele. Portio stark hypertrophisch. Muttermund durch beiderseitige Cervixrisse quer klaffend, Ectropium beider Lippen. Uterus anteflectirt, beweglich, stark vergrössert, über faustgross, von derber Consistenz, mit etwas unregelmässiger Oberfläche.

Operation: In Medullaranästhesie zunächst keilförmige Amputation beider hypertmphischen Muttermundslippen. Vordere :Kolporrhaphie. Hochschieben der Blase. Vorziehen des über faustgrossen, mit einigen Myomknötchen besetzten Uterus. Abbinden der Tuben zur Ausschaltung der Spermatica-Anastomosen. Resection eines grossen Keils aus dem Corpus uteri. Der Keil enthält mehrere kleine intramurale Myomkeime, einige subseröse Knötchen und ein submucöses Myom von der Grösse einer Walnuss. Aus den seitlichen Reststïcken des Uterus werden einige kleine intramurale Myome enucleirt. Die Uteruswand ist stark metritisch verdickt. Vereinigung der seitlichen, durch die Enucleation etwas unregelmässig gewordenen Schnittfächen durch zahlreiche Nähte. Die seitlichen Uterustheile enthalten keine Myome mehr, ebenso wenig die Cervix. Der neugebildete Uterus hat đie Grösse eines normalen Organs. Auf die Hinterfläche des neuen Cterus wird das vordere Peritoneum aufgenäht. Die Blutung während der Operation war nicht beträchtlich. Vaginale Fixation des neugebildeten Uterus. Hintere Kolporrhaphie und Dammplastik.

Reconvalescenz gestört durch bald nach der Operation einsetzende Temperatursteigerungen. Höchste Temperatur 40,00 am 4. Tage. Darauf langsamer Abfall, in der 2. und 3. Woche immer noch Temperatur bis $38,2^{\circ}$. Allgemeinbefinden dabei stets gut. Höchste Pulsfrequenz 108. Bräunlicher Fluor, durch Spülungen bald beseitigt. Erst in der 4. Woche normale Temperatur. Am 26. Tage nach dem Aufstehen nochmals einmalige. Steigerung auf 38,60 .

Entlassung am 31. Tage beschwerdefrei und in wesentlich gehobenem Allgemeinzustand. Der neugebildete Uterus ist von normaler Grösse und liegt gut gestreckt. Gutes Resultat der Scheidendammplastik.

Nachuntersuchung nach 1/4 Jahr: Wohlbefinden. Gutes plastisches Resultat. Uterus klein, gut gestreckt. Nur einmaliger geringer Blutabgang vor 8 Tagen.

\section{Fall 27. 1907/400. Fr. Si., 45 Jahre.}

Anamuese: 4 spontane Geburten, letzte vor 4 Jahren, Wochenbetten afebril. Seit 12 Jahren Vorfall der Scheide, der sich seit der letzten Geburt bedeutend verschlimmert hat. Ringbehandlung erfolglos. In deri letzten Jahren unregelmässig anteponirende, sehr starke und langdauernde Periode. Heftige Druckschmerzen im Leib. Patientin ist durch die starken Beschwerden jetzt arbeitsunfähig.

Status: Introitus klaffend. Alter Scheidendammriss. Die stark hypertrophische Portio liegt in der Vulva. Hochgradiger Descensus der vorderen und hinteren Vaginalwand. Halbfaustgrosse Cystocele, kleinere Rectocele. Der Uterus liegt in Mittelstellung; ist bis auf Faustgrösse verdickt, beweglich.

Operation: In Medullaranästhesie hohe Portioamputation. Vordere 
Kolporrhaphie. Hochschieben der Blase. Vorstülpen des faustgrossen Uteruskörpers. Präventive Ligirung der Tube und des Lig. rotundum jederseits. Excision eines grossen Keils aus dem Corpus, so dass die Corpusmucosa vollkommen entfernt wird. Vernähung der beiden seitlichen Uterustheile durch eine versenkte und eine oberflächliche Knopfnahtetage. Vaginale Fixation des neugebildeten Uterus. Hintere Kolporraphie und Perineoplastik.

Reconvalescenz völlig glatt. Keine Temperatursteigerung. Primäre Wundheilung. Patientin verlässt am 12. Tage das Bett.

Am 21. Tage Entlassung. Der neugebildete Uterus hat die Grösse eines normalen Organs und liegt gut gestreckt. Gutes Resultat der Scheidendammplastik. Wohlbefinden, keine Beschwerden.

Fall 28. 1907/495. Fr. St., 43 Jahre.

Anamnese: 2 normale Geburten, letzte vor $6 \frac{1}{2}$ Jabren. Seit 5 Jahren antepouirende und sehr starke Periode mit prä- und postmenstruellen Beschwerden. Im Intervall Fluor. Druckgefühl nach unten. Häufiger Urindrang. Ringbehandlung erfolglos.

Status: Hiatus vulvae. Descensus der Scheide. Cystocele. Breites massiges Collum. Ectropium. Ueber faustgrosser, druekempfindlicher, retrovertirt-flectirter Uterus.

Operation: In Aethernarkose Dilatation und Abrasio, die mässige Mengen normaler Schleimhaut zu Tage fördert. Darauf Amputation des hypertrophischen Collum. Vordere Kolporrhaphie. Ausgedehnte Auslösung der Blase und Hochschieben derselben. Eröffunng des Peritoneum und Vorleiten des Corpus uteri. Keilförmige Resection der mittleren $2 / 3$ des Uteruskörpers. Ziemlich starke Blutung. Vereinigung der seitlichen Uterusreste. Feste vaginale Fixation des neugebildeten Uterus. Hintere Kolporrbaphie und Dammplastik.

Reconvalescenz in der ersten Woche durch starke Reaction mit hohen Temperaturen gestört. Höchste Temperatur $40^{\circ}$ am 2. Tage. In der 3. Woche nach Verlassen des Bettes nochmalige vorübergehende Temperatursteigerung. Darnach glatte Reconvalescenz.

Am 29. Tage beschwerdefrei mit gutem plastischen Resultat entlassen.

Fall 29. 1907/513. Fr. M., 50 Jahre.

Anamnese: 5 spontane Geburten, letzte vor 13 Jahren. Seit mehreren Jahren Senkungsbeschwerden und Druckgefühl. Seit einem Jahre unregelmässige, manchmal sehr starke Blutung.

Status: Ziemliche Anämie. Klaffender Introitus. Alter Dammriss. Starker Descensus der vorderen und hinteren Vaginalwand. Uterus stark vergrössert, hart, beweglich, in Mittelstellung.

Operation: In Aethernarkose zunächst Probeabrasio mit geringem Ergebniss. Vordere Kolporraphie und Hochschieben der Blase. Eröffnung des Peritoneum. Vorziehen des metritisch verdickten Corpus. Beiderseitige Abbindung der Tuben zur Ausscbaltung der SpermaticaAnastomosen. Excision eines grossen Keils aus dem Corpus, wobei es zweifellos in Folge der seitlichen Unterbindung weniger stark blutet. Von der Corpusschleimhaut bleibt an dem linken Reststück ein Theil stehen. Vereinigung der seitlichen Theile zu einem ungefähr normal grossen Uterus, der vaginal fixirt wird. Hintere Kolporrhaphie und Dammplastik. 
Reconvalescenz glatt. Keine Temperatursteigerung. Gute Wundheilung. Patientin steht am 11. Tage auf.

Entlassung am 19. Tage. Wohlbefinden. Uterusstumpf von normaler Grösse, gut gestreckt. Scheide eng.

Fall 30. 1907/526. Fr. V., 41 Jabre.

Anamnese: 2 spontane Geburten, letzte vor 5 Jahren. Seit der letzten Geburt Vorfall. In der letzten Zeit unregelmässige und starke Periode. Vor 2 Jahren auswärts Prolapsoperation. Nach 1/2 Jahr totales Recidiv.

Status: Hiatus vulvae. Vor der Vulva das stark hypertrophische Collum mit Ectropium und Decubitalgeschwüren. Corpus retroflectirt, stark vergrössert, hart. Sondenlänge $81 / 2 \mathrm{~cm}$.

Operation: In Aethernarkose Amputation der hypertrophischen Portio. Vordere Kolporrhaphie, Abschieben der Blase. Nach Lösung der durch die vorige Operation entstandenen utero-vesicalen Peritonealverwachsungen wird das stark verdickte Corpus vorgezogen. Keilresection aus dem Corpus, von welchem nur ein kleiner Theil übrig bleibt. Starke Blutung. Uterusneoplastik. Auf die Hinterfläche des neugebildeten Stumpfs wird das Peritoneum aufgenäht. Vaginale Fixation des Stumpfs. Versehluss der Kolporrhaphie.

Reconvalescenz zunächst glatt. Vom 7.--11. Tage Temperatursteigerung mit starker Secretion. Vom 12. Tage ab normale Temperatur und glatte Heilung. lassen.

Am 22. Tage beschwerdefrei mit gutem plastischen Resultat ent- 